\title{
Effect of Feedback on the Capacity of Discrete Additive Channels with Memory $^{\dagger}$
}

\author{
Fady Alajaji and Tom Fuja \\ Department of Electrical Engineering and Institute for Systems Research \\ University of Maryland, College Park, MD 20742, USA
}

\section{Overview}

We consider discrete channels with arbitrary additive noise. Note that such channels need not be memoryless; in general, they have memory. We show that feedback does not increase the capacity of such channels. The same result is also shown for a larger class of channels to which additive channels belong, the class of discrete symmetric channels with memory. These channels have the property that their inf-information rate is maximized for equally likely iid input processes. Finally, we impose cost constraints on the input of the additive channels, rendering them non-symmetric. In this case, we show that feedback can increase their capacity-cost function.

\section{Additive Channels}

Consider a channel with alphabet $A=\{0,1, \ldots, q-1\}$, described by: $Y_{n}=X_{n} \oplus Z_{n}$, for $n=1,2,3, \ldots$, where the addition is modulo $q$ and $X_{n}, Z_{n}$ and $Y_{n}$ are respectively the input, noise and output of the channel. We assume that the input and noise sequences are independent $\left(\left\{X_{n}\right\} \perp\left\{Z_{n}\right\}\right)$ and that the noise process $\left\{Z_{n}\right\}$ is an arbitrary process (nonstationary non-ergodic in general).

We assume that the channel is accompanied by a noiseless, delayless feedback channel with large capacity. The receiver uses the feedback channel to inform the transmitter what letters were actually received; these letters are received at the transmitter before the next letter is transmitted, and therefore can be used in choosing the next transmitted letter.

The capacity with no feedback (with feedback) denoted by $C_{N F B}\left(C_{F B}\right)$, is the supremum of all rates for which there exist nonfeedback (feedback) block codes with vanishing probability of error. In [1], Verdú and Han derived a general formula for the (nonfeedback) capacity of arbitrary channels: $C_{N F B}=\sup _{X^{n}} \underline{I}\left(X^{n} ; Y^{n}\right)$, where $X^{n}=\left(X_{1}, X_{2}, \ldots, X_{n}\right)$ is the input vector and $Y^{n}$ is the corresponding output vector. The symbol $\underline{I}\left(X^{n} ; Y^{n}\right)$ is the inf-information rate between $X^{n}$ and $Y^{n}$ and is defined as the liminf in probability [1] of $\frac{1}{n} i_{X^{n} Y^{n}}\left(X^{n} ; Y^{n}\right)=\frac{1}{n} \log _{2} \frac{P_{Y^{n} \mid X^{n}\left(Y^{n} \mid X^{n}\right)}}{P_{Y^{n}\left(Y^{n}\right)}}$.

Theorem 1 Feedback does not increase the capacity of discrete channels with arbitrary additive noise [2]:

$$
C_{F B}=C_{N F B}=\log _{2}(q)-\bar{H}\left(Z^{n}\right),
$$

where $\bar{H}\left(Z^{n}\right)$ is the sup-entropy rate of the additive noise process $\left\{Z_{n}\right\}$, which is the limsup in probability [1] of $\frac{1}{n} \log _{2} \frac{1}{P_{Z^{n}}\left(Z^{n}\right)}$.

\footnotetext{
${ }^{\dagger}$ Supported in part by NSF grant NCR-8957623; also by the NSF Engineering Research Centers Program, CDR-8803012. Additional travel support is provided to the first author by the Graduate School at the University of Maryland.
}

\section{Remarks}

(i) If the noise process is stationary, then its sup-entropy rate is equal to the supremum over the entropies of almost every ergodic component of the stationary noise.

(ii) If the noise process is stationary ergodic, then its supentropy rate is equal to its entropy rate.

\section{Symmetric Channels}

Definition Consider the channel given by: $Y_{n}=f\left(X_{n}, Z_{n}\right)$, for $n=1,2,3, \ldots$ where $X_{n}, Z_{n}$ and $Y_{n}$ are as defined above, $\left\{X_{n}\right\} \perp\left\{Z_{n}\right\}$, and $f: \mathcal{X} \times \mathcal{Z} \rightarrow \mathcal{Y}$, is a mapping from the cartesian product of the input alphabet $\mathcal{X}$ and the noise alphabet $\mathcal{Z}$ into the output alphabet $\mathcal{Y}$. We assume that the sets $\mathcal{X}, \mathcal{Z}$ and $\mathcal{Y}$ are all finite. We say that such a channel is symmetric if the following conditions hold:

1. $|\mathcal{X}|=|\mathcal{Y}|=|\mathcal{Z}|=q$; i.e., the input, output and noise alphabets have same cardinality.

2. Given the input $x$, the function $f(x,$.$) is one-to-one; i.e.$ for all $x \in \mathcal{X}, f(x, z)=f(x, \tilde{z}) \Longrightarrow z=\tilde{z}$.

3. $f^{-1}(\cdot)$ exists such that $z=f^{-1}(x, y)$ and given the output $y, f^{-1}(\cdot, y)$ is one-to-one; i.e. for all $y \in \mathcal{Y}, f^{-1}(x, y)=$ $f^{-1}(\tilde{x}, y) \Longrightarrow x=\tilde{x}$.

Theorem 2 Feedback does not increase the capacity of discrete symmetric channels:

$$
C_{F B}=C_{N F B}=\log _{2}(q)-\bar{H}\left(Z^{n}\right) .
$$

\section{Additive Channels with Input Constraints}

We incorporate average cost constraints on the input sequences of the additive noise channel. In [3], we demonstrate that in the case where the additive noise is a binary stationary mixing homogeneous Markov process, feedback can increase the capacity-cost function of this channel. This is achieved by establishing a tight upper bound to the capacity-cost function of the channel with no feedback $\left(C_{N F B}(\beta)\right)$ and a lower bound to the capacity-cost function with feedback $\left(C_{F B}(\beta)\right)$. The upper bound to $C_{N F B}(\beta)$ is nothing but the dual of the Wyner-Ziv lower bound to the rate distortion function of stationary ergodic sources. The lower bound to $C_{F B}(\beta)$ is then shown numerically to exceed the upper bound to $C_{N F B}(\beta)$.

\section{REFERENCES}

[1] S. Verdú and T. S. Han, "A General Formula for Channel Capacity", IEEE Trans. Information Theory, to appear.

[2] F. Alajaji, "Feedback Does Not Increase the Capacity of Discrete Channels with Additive Noise", submitted to IEEE Trans. Information Theory.

[3] F. Alajaji and T. Fuja, "Feedback Can Increase the Capacity-Cost Function of Discrete Channels with Memory", Proc. of the Conf. Inform. Sci. and Syst., Princeton University, Princeton, NJ, March 1994. 